\title{
Effects of Initialization Conditions on Erasability of Phase Change Optical Disks
}

\author{
Jia-Reuy Liu and Han-Ping D. Shieh \\ Institute of Electro-Optical Engineering, National Chiao Tung University, Hsinchu, Taiwan, R.O.C.
}

\begin{abstract}
Effects of initialization conditions of initialization power and number of initialization cycles on the recording properties of phase change optical disks were studied. It is found that initialization conditions affect erasability but have little effect on carrier-to-noise ratio of phase change optical disks. The initialization conditions also affect bias power margin. Erasability of more than $20 \mathrm{~dB}$ and carrier-to-noise ratio of $54 \mathrm{~dB}$ with $4 \mathrm{~mW}$ bias power margin were obtained by applying $6 \mathrm{~mW}$ of initialization power and 5 initialization cycles.
\end{abstract}

\section{INTRODUCTION}

Erasable phase change optical disks have many advantages as recording media such as direct overwriting, compatibility with $\mathrm{CD}$ and no need of an external magnetic bias field. Writing and erasing on phase change disks are achieved by phase transformation between amorphous and crystalline state. In writing, the recording layer is heated over it's melting point and then quenched to amorphous state; in erasing, the amorphous marks are annealed to crystalline state. Usually the written marks can not be completely erased in overwriting [1], which results in residual signal. Hence, erasability is an important parameter to assess the performance of erasable phase change optical disks. In this work, the effects of initialization conditions on erasability, carrier-to-noise ratio, and bias power margin of phase change optical disks were studied.

\section{EXPERIMENTS}

Typical erasable phase-change disks are couposed of magnetron-sputter deposited thin-film layers. Recording layer, $\mathrm{Ge}_{20} \mathrm{Te}_{53} \mathrm{Sb}_{27}$, is sandwiched between upper and lower $\mathrm{ZnS}-\mathrm{SiO}_{2}$ dielectric layers; and $\mathrm{Al}$ is employed as a reflective layer. To minimize crystalline grains growing around amorphous marks, a rapid cooling structure [2] using a rather thin upper dielectric layer was applied.

The effect of initialization conditions on erasability was investigated by a dynamic tester with a $780 \mathrm{~nm}$ wavelength laser diode and a $0.55 \mathrm{NA}$ (numerical aperture) objective lens. Erasability is defined by

Erasability $=$ CNR of $f_{1}$ - CNR of residual of $f_{1}$ overwritten by $f_{2}$

where $f_{1}$ and $f_{2}$ represent writing and overwriting signals,

Manuscript received June 25, 1997

Jia-Reuy Liu, Author, u8224502@cc.nctu.edu.tw

Han-Ping D. Shieh, hpshieh@cc.nctu.edu.tw respectively. Because the as-deposited phase change media are in amorphous state, the data tracks must first be initialized to crystalline state by irradiating dc erase power $\mathrm{P}_{\mathrm{e}}$ before recording. In initialization process, "dc erase power $\mathrm{P}_{\mathrm{e}}$ " was denoted by "initialization power $\mathrm{P}_{i}$ ". Initialization power $P_{i}$ of 3 to $7 \mathrm{~mW}$ and 1 to 100 initialization cycles were used to study the optimal initialization conditions.

After the initialization, the disks were characterized as following: first, an $f_{1}$ signal was written on given tracks and the carrier and noise levels were derived. Then, an $\mathrm{f}_{2}$ signal was applied to overwrite these tracks and the carrier and noise levels were also derived. In addition, the carrier and noise levels of the residual $f_{1}$ signal were also measured. Erasability and CNR were thus calculated from these data.

\section{RESULTS AND DISCUSSION}

\section{A. Requirements of phase change optical disks}

Conferring with the specification of commercial phase change optical disks [3], CNR must be above $46 \mathrm{~dB}$ and erasability must be above $18 \mathrm{~dB}$ at the read power of $1 \mathrm{~mW}$. Because the read power needs to be raised to $1.5 \mathrm{~mW}$ for stable focusing and tracking in this study, hence the required CNR must be higher than $49 \mathrm{~dB}$.

Initialization power $P_{i}$ of $5 \mathrm{~mW}$ were applied to \#134 disk with structure of substrate $/ 140 \mathrm{~nm} \mathrm{ZnS}-\mathrm{SiO}_{2} / 25 \mathrm{~nm}$ $\mathrm{Ge}_{20} \mathrm{Te}_{53} \mathrm{Sb}_{27} / 37 \mathrm{~nm} \mathrm{ZnS-SiO} / 50 \mathrm{~nm}$ Al. It was found that recording at $\mathrm{P}_{\mathrm{W}}$ of $11 \mathrm{~mW}$ and $\mathrm{P}_{\mathrm{b}}$ of $5 \mathrm{~mW}$ allowed CNR to reach $49 \mathrm{~dB}$, as shown in Table $I$. Moreover, erasability of high-frequency signal to be overwritten by low-frequency signal was more than that of low-frequency signal to be overwritten by high-frequency signal, as shown in

TABLE I: DEPENDENCE OF ERASABILITY OF \#134 DISK ON $\mathbf{f}_{1}$ AND $\mathbf{f}_{\mathbf{2}}$ (a) linear speed: $5.5 \mathrm{~m} / \mathrm{s}$

\begin{tabular}{ccccc}
\hline \multicolumn{6}{c}{ (a) linear speed: $5.5 \mathrm{~m} / \mathrm{s}$} \\
$\begin{array}{c}\mathrm{f}_{1} \\
(\mathrm{MHz})\end{array}$ & $\begin{array}{c}\mathrm{f}_{2} \\
(\mathrm{MHz})\end{array}$ & $\begin{array}{c}\text { CNR of } \mathrm{f}_{1} \\
(\mathrm{~dB})\end{array}$ & $\begin{array}{c}\text { CNR of } \mathrm{f}_{2} \\
(\mathrm{~dB})\end{array}$ & $\begin{array}{c}\text { Erasability } \\
(\mathrm{dB})\end{array}$ \\
\hline 1.5 & 4 & 52 & 49 & 20 \\
4 & 1.5 & 49 & 51 & 25 \\
\hline
\end{tabular}

(b) linear speed: $11 \mathrm{~m} / \mathrm{s}$

\begin{tabular}{|c|c|c|c|c|}
\hline $\begin{array}{c}\mathbf{f}_{\mathfrak{l}} \\
(\mathrm{MHz})\end{array}$ & $\begin{array}{c}f_{2} \\
(\mathrm{MHz})\end{array}$ & $\begin{array}{l}\text { CNR of } f_{i} \\
\text { (dB) }\end{array}$ & $\begin{array}{c}\text { CNR of } f_{2} \\
\text { (dB) }\end{array}$ & $\begin{array}{c}\text { Erasability } \\
\text { (dB) }\end{array}$ \\
\hline 3.4 & 9 & 52 & 49 & 10 \\
\hline 9 & 3.4 & 49 & 54 & 20 \\
\hline \multicolumn{3}{|c|}{$\begin{array}{l}P_{W}=11 \mathrm{~mW}, P_{b}=5 \mathrm{~mW}, P_{r}=1.5 \mathrm{~mW} \\
\text { and } P_{i}=5 \mathrm{~mW}\end{array}$} & Pr ${ }^{\mathrm{Pb}}$ & $\mathrm{Pb}$ \\
\hline
\end{tabular}


Table I(a) and (b). Compared overwriting at different linear speed, which corresponds to data tracks at different radius on the disks rotating at a constant angular velocity (CAV), Table I reveals that erasability decreases with increasing linear speed. Therefore, the testing parameters were set as following: low-frequency signal was first written, then high-frequency signal was applied to overwrite at linear speed of $11 \mathrm{~m} / \mathrm{s}$. As the erasability under these testing parameters reached $18 \mathrm{~dB}$, then erasability under other testing conditions of Table I shall also satisfy the erasability of more than $18 \mathrm{~dB}$.

\section{B. Effect of initialization power on erasability}

The initialization results of \#134 disk by different $P_{i}$ were shown in Table II. The measured erasability was only $15 \mathrm{~dB}$ at $\mathrm{P}_{i}$ of $3 \mathrm{~mW}$, but raised up to $20 \mathrm{~dB}$ when $\mathrm{P}_{i}$ was increased to $5 \mathrm{~mW}$ at linear speed of $5 \mathrm{~m} / \mathrm{s}$, as shown in Table II(a). However, the erasability was unable to reach $18 \mathrm{~dB}$ at linear speed of $11 \mathrm{~m} / \mathrm{s}$, as shown in Table II (b). It was also found that CNR was more than $48 \mathrm{~dB}$ and did not change with $\mathrm{P}_{\mathrm{i}}$, as shown in Table II(a) and (b).

Because the erasability of \#134 disk was less than $18 \mathrm{~dB}$ at linear speed of $11 \mathrm{~m} / \mathrm{s}$, a series of disks with different structure were made to further investigate their erasability at high linear speed. The \#175 disk with structure of substrate/140nm ZnS-SiO $/ 25 \mathrm{~nm} \mathrm{Ge} 20 \mathrm{Te}_{53} \mathrm{Sb}_{27} / 25 \mathrm{~nm} \mathrm{ZnS-}$ $\mathrm{SiO}_{2} / 40 \mathrm{~nm} \mathrm{Al}$ was found to possess adequate overwriting characteristics at linear speed of $11 \mathrm{~m} / \mathrm{s}$. The dependence of its erasability on $P_{i}$, as shown in Fig. 1 , revealed that erasability was above $18 \mathrm{~dB}$ when $P_{\mathrm{i}}$ was from 5 to $7 \mathrm{~mW}$.

From above measurements, we find that initialization power affects erasability but does not affect CNR. To further examine the dependence of erasability on $\mathrm{P}_{\mathrm{i}}$, equation ( $\mathrm{l}$ ) is redefined by

$$
\begin{aligned}
\text { Erasability } & =\left(C_{W}-N_{W}\right)-\left(C_{O W}-N_{O W}\right) \\
& =\left(C_{W}-C_{O W}\right)-\left(N_{W}-N_{O W}\right)
\end{aligned}
$$

where $C_{W}$ and $N_{W}$ are carrier and noise levels of $f_{1}$ before been overwritten, respectively; $C_{O W}$ and $N_{O W}$ are carrier and noise levels of $f_{1}$ after been overwritten by $f_{2}$, respectively. Thus, the term $\left(C_{W}-N_{W}\right)$ represents $C N R$ of $f_{1}$, while $\left(C_{O W}\right.$ - $N_{\text {ow }}$ ) represents the residual of $f_{1}$ after been overwritten.

TABLE II: DEPENDENCE OF ERASABLITY ON $P_{1}$

(a) linear speed: $5.5 \mathrm{~m} / \mathrm{s}, \mathrm{f}_{1}=4 \mathrm{MHz}, \mathrm{f}_{2}=1.5 \mathrm{MHz}$

\begin{tabular}{cccc}
\hline Initialization power $\mathrm{P}_{\mathrm{i}}(\mathrm{mW})$ & 3 & 4 & 5 \\
\hline CNR of $\mathrm{f}_{1}(\mathrm{~dB})$ & 50 & 50 & 51 \\
CNR of $\mathrm{f}_{2}(\mathrm{~dB})$ & 48 & 48 & 49 \\
Erasability $(\mathrm{dB})$ & 15 & 17 & 20 \\
\hline
\end{tabular}

\begin{tabular}{cccc}
\hline & 15 & 17 & 20 \\
\hline (b) linear speed: $11 \mathrm{~m} / \mathrm{s}, \mathrm{f}_{1}=3 \mathrm{MHz}, \mathrm{f}_{2}=8 \mathrm{MHz}$ & \\
\hline Initialization power $\mathrm{P}_{\mathrm{i}}(\mathrm{mW})$ & 3 & 4 & 5 \\
\hline CNR of $\mathrm{f}_{1}(\mathrm{~dB})$ & 54 & 54 & 55 \\
CNR of $\mathrm{f}_{2}(\mathrm{~dB})$ & 48 & 48 & 48 \\
Erasability $(\mathrm{dB})$ & 12 & 11 & 13 \\
\hline
\end{tabular}

$P_{W}=11 \mathrm{~mW}$ and $P_{b}=5 \mathrm{~mW}$

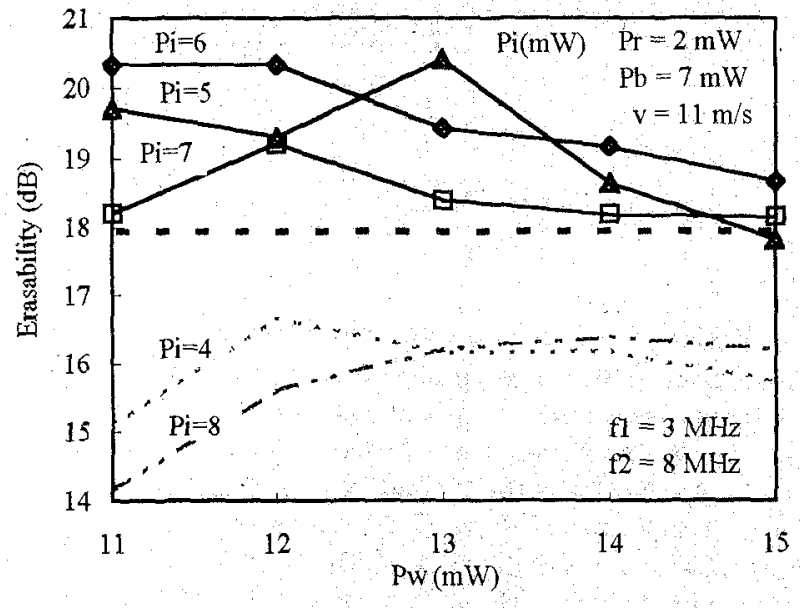

Fig. 1 Lependence of erasability of $\# 175$ disk on $P_{i}$ and $P_{w}$. Each data track was initialized 5 cycles by $P_{i}$.

Derived from the measured data, we found that erasability was proportional to $\left(\mathrm{C}_{\mathrm{W}}-\mathrm{C}_{\mathrm{OW}}\right)$ but almost independent of ( $\left.\mathrm{N}_{\mathrm{W}}-\mathrm{N}_{\mathrm{OW}}\right)$. After further inspection, the dependence of erasability on $\mathrm{C}_{\mathrm{W}}$ or $\mathrm{C}_{\mathrm{OW}}$, plotted in Fig. 2(a) and (b), which showed that initialization power affected $\mathrm{C}_{\mathrm{O}}$ w but had little effect on $C_{W}$. Compared Fig. 1 with Fig. 2, erasability is inversely proportional to $\mathrm{C}_{\mathrm{OW}}$, but not related to $C_{W}$, implying that $P_{i}$ affects erasability of the phase change disk by its residual signal level. The effects of initialization power on erasability were found not only in \#175 disk, but also in other phase change optical disks.

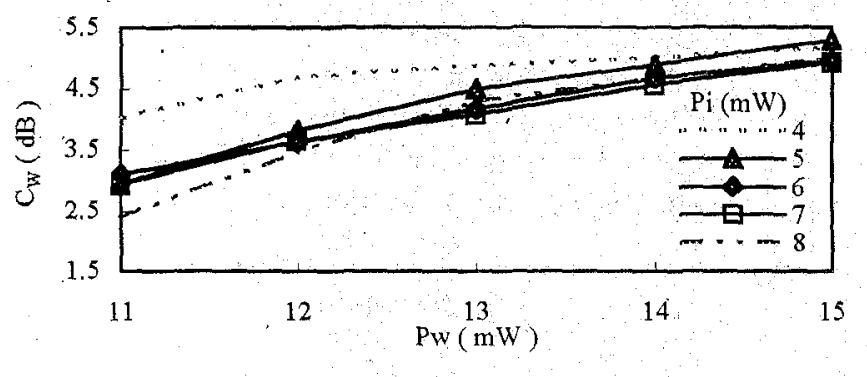

(a)

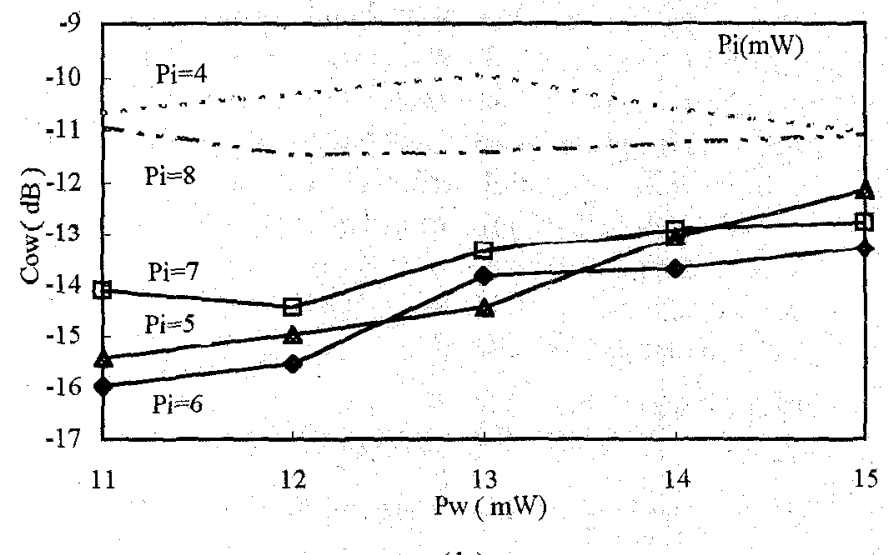

(b)

Fig. 2 Dependence of carrier levels (a) $C_{W}$ and (b) $C_{0 w}$ on $P_{i}$ and $P_{W}$ 
The variation of erasability might be caused by disk non-repeated runout, which could result in incomplete initialization. However, the full-width-at-half-maximum (FWHM) of the laser spot is 0.85 um and the tracking offset of the optical stylus is $0.1 \mathrm{um}$, so the optical stylus of $0.85 \pm 0.1 \mathrm{um}$ FWHM could cover a 0.5 um groove width of the disks during initialization. Besides, CNR of writing and overwriting signals were both more than $52 \mathrm{~dB}$. Therefore, the disk runout was not the main cause of the variation of erasability. It was suggested that the variant structures of the crystalline states with different $P_{i}$ might be one of the main reasons. Other factors such as the number of initialization cycles might also affect erasability.

\section{Effect of number of initialization cycles on erasability}

The number of initialization cycles $\mathrm{N}_{\mathrm{i}}$ was varied from 1 to 100 in the initialization process to study whether insufficient erasability was caused by incomplete crystallization. As shown in Fig. 3 , when $P_{i}=6 \mathrm{~mW}$, the erasability was found to be almost all above $18 \mathrm{~dB}$ except the data tracks initialized by only one time. Moreover, we found that more than 5 initialization cycles could not increase the erasability further. Consequently, the variation of erasability was not caused by incomplete initialization. Under the optimized initialization conditions of $\mathrm{P}_{\mathrm{i}}$ of $6 \mathrm{~mW}$ and $\mathrm{N}_{\mathrm{i}}$ of 5 cycles, CNR was more than $54 \mathrm{~dB}$ at 3 and $8 \mathrm{MHz}$ writing frequency, which corresponded to $1.2 \mathrm{um}$ and $0.63 \mathrm{um}$ mark length, respectively, and bias power margin was maximized as $\mathbf{4}$ mW, as shown in Fig. 4.

Hence, the initialization power and the number of initialization cycles should be chosen to make phase change disks with adequate erasability. However, the optical stylus of our dynamic tester is of circular shape in contrast to the elongated shape used in disk initializers[4], as shown in Fig. 5 . The energy generated by the elongated beam of 600 $\mathrm{mW} / 100 \mathrm{um} \times 2 \mathrm{um}$ with overlap ratio of $80 \%$ is close to that by the circular beam of $6 \mathrm{~mW} / 2 \mathrm{um}^{2}$ with $\mathrm{N}_{\mathrm{i}}$ of 5 cycles at the same linear speed in initialization process. Hence, the initialization effects of circular stylus with $\mathrm{N}_{i}$ cycles is comparable to that of disk initializers. The similarity of initialization effect may be caused by generating the similar

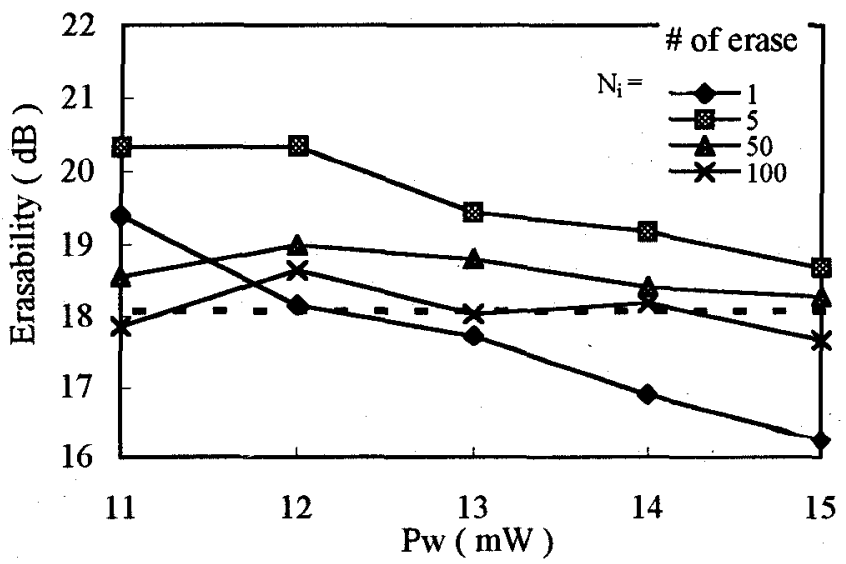

Fig. 3 Effect of the number of initialization cycles $\mathrm{Ni}$ on erasability.

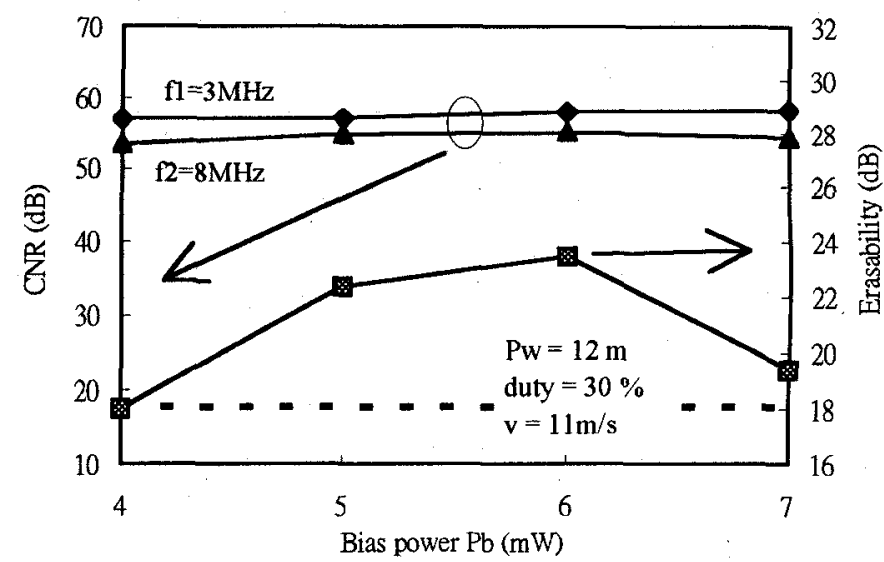

Fig. $4 \mathrm{CNR}$ and erasability of phase change disks. The phase change disk was initialized by $P_{i}=6 \mathrm{~mW}$ for 5 cycles.

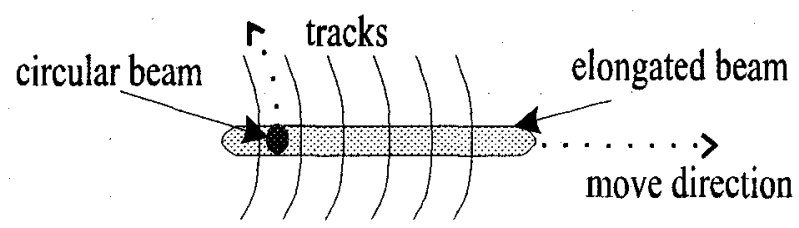

Fig. 5 Elongated beam and circular beam irradiated on optical disks

crystalline size during initialization by the same energy level. To further reveal the erasability on phase transformation and other causes, we shall study crystalline grains resulted by initialization processes, and it is the subject to be reported in near future.

\section{CONCLUSION}

The initialization process affected erasability of phase change disks, but had little effect on CNR. The consequence of initialization process of phase change disks was primarily on residual carrier level change but little on other signal levels. The initialization power and the number of initialization cycles should be properly chosen to make phase change disks with adequate erasability. Under the optimized initialization conditions of $P_{i}$ of $6 \mathrm{~mW}$ and $N_{i}$ of 5 cycles, CNR of $54 \mathrm{~dB}$ at data frequency of 3 and $8 \mathrm{MHz}$, and bias power margin of $4 \mathrm{~mW}$ were obtained at linear speed of $11 \mathrm{~m} / \mathrm{s}$.

\section{ACKNOWLEDGMENT}

The work was supported by the National Science Council, the Republic of China, under Contract No. NSC 86-2622-L009-002.

\section{REFERENCES}

[1] T. Ishuda, S. Ohara, N. Akahira, T. Ohta, and T. Yoshida, "Overwriting characteristics in phase change disk," Jpn. J. Appl. Phys., vol. 28, Suppl. 28-3, pp. 129-133, 1989.

[2] T. Ohta, K. Inoue, M. Uchida, K. Yoshioka, T. Akiyama, S. Furukawa, K. Nagata, and S. Nakamura, "Phase change disk media having rapid cooling structure,"Jpn. J. Appl. Phys., vol. 28, Suppl. 28-3, pp. 123-128, 1989.

[3] Matsushita PD specification, 1996.

[4] Hitachi phase change disk initializer specification, 1996 